\title{
What level of friction guarantees adhesion in a complete contact?
}

\author{
D A Hills* and D Dini \\ Department of Engineering Science, University of Oxford, Oxford, UK
}

\begin{abstract}
The Williams' solution for the state of stress near the apex of an elastic wedge is used to study the traction distribution adjacent to the tip of a complete contact. Assuming that the surfaces remain in contact during loading, the coefficient of friction needed to ensure local adhesion is found. This is compared with that predicted by an analysis of an elastic rectangle clamped between elastic halfplanes and is shown to be consistent. The results are appropriate to all complete contacts between elastically similar components.
\end{abstract}

Keywords: coefficient of friction, asymptotes, adhesion

\section{INTRODUCTION}

The intention in this brief note is to derive a simple result for the minimum coefficient of friction needed to ensure adhesion of a complete contact in the neighbourhood of the contact edge, for contact between elastically similar bodies. The result has wide-ranging applicability but has obvious application to the performance of complete contacts potentially undergoing fretting fatigue.

The inset of Fig. 1 depicts a general plane contact, which may have any shape and be multiply connected, as indicated by the presence of the cavity, but must be 'complete' in the sense that the contact face is flat, with discontinuities in the contact profile at the contact edges. It is subject to a normal force $P$, possibly a moment $M$ and a shearing force $Q$, and any of these may vary in time in an arbitrary way. The state of stress adjacent to a slipping corner for such a problem has been studied in detail by Gdoutos and Theocaris [1], but the question that is asked here is: 'What is the minimum value of the coefficient of friction to guarantee adhesion, in the neighbourhood of the contact corner A?' The most obvious way to approach the problem is to solve for the interfacial contact pressure distribution and the shear traction distribution. These functions will, in general, clearly be a function of time, and the problem may therefore require a complex numerical procedure, possibly using the finite element method,

The MS was received on 11 September 2003 and was accepted after revision for publication on 2 April 2004.

* Corresponding author: Department of Engineering Science, University of Oxford, Parks Road,Oxford OX13PJ,UK.E-mail: david.hills@eng.ox. ac.uk and the possibility of local slipping checked by determining the ratio $\sigma_{r \theta} / \sigma_{\theta \theta}$ along the interface. All slip will be avoided if this ratio is everywhere less than the coefficient of friction, $f$. This detailed calculation may not, however, be necessary. Suppose for the time being that the contact problem has been solved, assuming adhered conditions at point $\mathrm{A}$. In that case, because it has also been assumed that the two bodies are elastically similar, the stress state in the neighbourhood of A may be found from a consideration of classical Williams notch asymptotics [2], by taking the included angle within the material to be $\pi+\phi$. The conventional solution to such a problem decomposes the eigensolutions into a symmetric component and an antisymmetric component with respect to the centre-line (B-B in Fig. 1) [3], which, in fracture mechanics nomenclature would be thought of as mode I loading and mode II loading respectively. If the corresponding multipliers, or generalized stress intensity factors, are given by $K_{\mathrm{I}}$ and $K_{\mathrm{II}}$ the mode mixity present in the actual problem would yield a matrix of the form

$$
\left\{\begin{array}{c}
K_{\mathrm{I}} \\
K_{\mathrm{II}}
\end{array}\right\}=\left[\begin{array}{lll}
? & ? & ? \\
? & ? & ?
\end{array}\right]\left\{\begin{array}{c}
P \\
Q \\
M
\end{array}\right\}
$$

The question marks could be revealed only by evaluating the full problem. This may not, however, be necessary. The total state of stress in the bodies in the neighbourhood of A may be written as

$$
\sigma_{i j}(r, \theta)=K_{\mathrm{I}} r^{\lambda_{\mathrm{I}}-1} f_{i j}^{\mathrm{I}}(\theta)+K_{\mathrm{II}} r^{\lambda_{\mathrm{II}}-1} f_{i j}^{\mathrm{II}}(\theta)
$$

where $\lambda_{k}$ is the eigenvalue and $f_{i j}^{k}(\theta)$ the corresponding spatial distribution of stresses, or eigenfunction, for mode $k$ loading $(k=\mathrm{I}$, II). For $\phi<\pi$, which is true for all punches, it is known that $[\mathbf{2}, \mathbf{3}] \lambda_{\mathrm{I}}-1<\lambda_{\text {II }}-1<0$. 


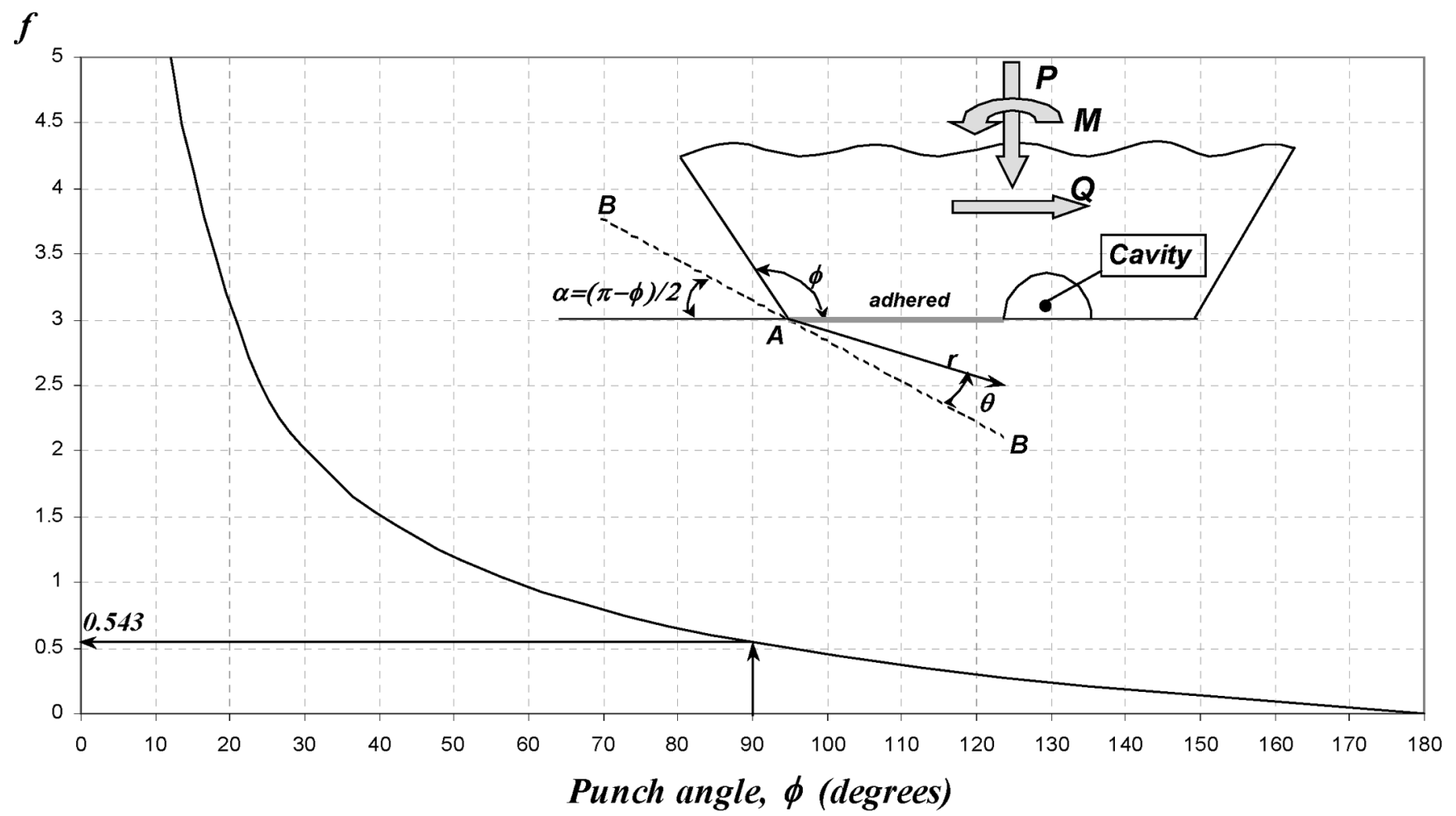

Fig. 1 Geometry of the general complete problem to be studied and the minimum friction coefficient that ensures adhesion as a function of the punch angle, $\phi$

It follows that the state of stress is always singular and, furthermore, that the mode I component of loading will always dominate the mode II component, as $r \rightarrow 0$, although, if $\phi$ is very large (which is unlikely in practice) the contribution from the latter may be important if $K_{\mathrm{II}} \gg K_{\mathrm{I}}$. Indeed, if $\phi<77.4^{\circ}$ the mode II eigensolution is bounded. Therefore, in subsequent discussion, without loss of generality, attention may be focused on the mode I component of loading alone.

Explicitly, the state of stress at small values of $r$ may be written as*

$$
\begin{aligned}
\left\{\begin{array}{c}
\sigma_{\theta \theta} \\
\sigma_{r r} \\
\sigma_{r \theta}
\end{array}\right\}= & K_{\mathrm{I}} r^{\lambda_{\mathrm{I}}-1}\left\{\begin{array}{c}
f_{\theta \theta}^{\mathrm{I}}(\theta, \alpha) \\
f_{r r}^{\mathrm{I}}(\theta, \alpha) \\
f_{r \theta}^{\mathrm{I}}(\theta, \alpha)
\end{array}\right\} \\
= & \frac{K_{\mathrm{I}} r^{\lambda_{\mathrm{I}}-1}}{\left(\lambda_{\mathrm{I}}+1\right)+\Gamma_{\mathrm{I}}\left(1-\lambda_{\mathrm{I}}\right)} \\
& \times\left(\left\{\begin{array}{c}
\left(\lambda_{\mathrm{I}}+1\right) \cos \left[\left(1-\lambda_{\mathrm{I}}\right) \theta\right] \\
\left(3-\lambda_{\mathrm{I}}\right) \cos \left[\left(1-\lambda_{\mathrm{I}}\right) \theta\right] \\
\left(1-\lambda_{\mathrm{I}}\right) \sin \left[\left(1-\lambda_{\mathrm{I}}\right) \theta\right]
\end{array}\right\}\right. \\
& \left.\left.+\Gamma_{\mathrm{I}}\left(1-\lambda_{\mathrm{I}}\right)\left\{\begin{array}{c}
\cos \left[\left(1+\lambda_{\mathrm{I}}\right) \theta\right] \\
-\cos \left[\left(1+\lambda_{\mathrm{I}}\right) \theta\right] \\
\sin \left[\left(1+\lambda_{\mathrm{I}}\right) \theta\right]
\end{array}\right\}\right)\right]
\end{aligned}
$$

\footnotetext{
* Here the notation used by Filippi et al. [4] is employed.
}

where the only non-trivial solution to the mode I eigenvalue is given by

$$
\sin \left(\lambda_{\mathrm{I}} q \pi\right)+\lambda_{\mathrm{I}} \sin (q \pi)=0
$$

and

$$
\begin{aligned}
& q=\frac{2 \pi-2 \alpha}{\pi} \\
& \Gamma_{\mathrm{I}}=-\frac{\sin \left[\left(1-\lambda_{\mathrm{I}}\right) q \pi / 2\right]}{\sin \left[\left(1+\lambda_{\mathrm{I}}\right) q \pi / 2\right]}
\end{aligned}
$$

Consider, now, the stress components present along the interface, i.e. along $\alpha=(\pi-\phi) / 2$. The ratio of the traction components is given by

$$
\frac{\sigma_{r \theta}}{\sigma_{\theta \theta}}=\frac{\left(1-\lambda_{\mathrm{I}}\right) \sin \left[\left(1-\lambda_{\mathrm{I}}\right) \alpha\right]+\Gamma_{\mathrm{I}}\left(1-\lambda_{\mathrm{I}}\right) \sin \left[\left(1+\lambda_{\mathrm{I}}\right) \alpha\right]}{\left(\lambda_{\mathrm{I}}+1\right) \cos \left[\left(1-\lambda_{\mathrm{I}}\right) \alpha\right]+\Gamma_{\mathrm{I}}\left(1-\lambda_{\mathrm{I}}\right) \cos \left[\left(1+\lambda_{\mathrm{I}}\right) \alpha\right]}
$$

If the coefficient of friction exceeds this value, a pointwise application of Amontons' law of friction requires the two bodies to be in adhesion in this region. This function is plotted in Fig. 1. It shows that, when the value of $\phi$ is large and, incidentally, when the order of the singularity approaches its strongest value, a very small coefficient of friction will ensure adhesion. As $\phi$ is reduced, the required value of the coefficient of friction is increased, so that for a square ended punch it is equal to $f=0.543$. In one of the very few analytical treatments of complete contact between elastically similar bodies, 
Khadem and O'Connor [5] examined the contact between a rectangular punch sandwiched between two elastic half-planes. They concluded that, remote from the edge, the coefficient of friction would need to be 0.5 to inhibit slip remote from the corners, and this result is clearly complementary to theirs. For problems in which the angle at the contact edge is less than $\pi / 2$ the coefficient of friction required continues to increase; it exceeds unity at about $58^{\circ}$ and increases monotonically with decreasing $\phi$, so that it is impossible to maintain adhesion for a slender 'finger'. It is emphasized that this result places no restriction whatever on the nature of the loading, save that the contact remain in adhesion right up to the contact edge.

It should be borne in mind that these results apply only to the possibility that slip emanates from the contact edge, and that the possibility that slip starts from an interior point within the contact, where the asymptote does not apply, has not been addressed. Nevertheless, experience in analysing a wide range of contacts has shown that slip invariably starts at the edge, but the possibility of finding a counter-example cannot be excluded. Finally, in a real problem there will always be some minute edge radius, possibly caused by local plasticity; provided that this is small compared with the region in which the mode I eigensolution dominates, the results derived will continue to hold.

\section{CONCLUSION}

In this brief note the authors have found the minimum coefficient of friction that must be present at a contact edge to ensure that adhesion is achieved. If the punch is 'hard', and this is treated as meaning 'relatively rigid' by comparison with the half-plane, an equivalent calculation to that described may be followed using the Bogy [6] solution for the stress state adjacent to the apex of bounded wedges. Now, one wedge becomes the edge of the contacting body and is made perfectly rigid, while the other is given an internal angle of $\pi \mathrm{rad}$, to become a half-plane. With the exception of the special case when the half-plane is incompressible (Poisson's ratio $\nu=\frac{1}{2}$, in which case the two eigensolutions have the same strength) a similar argument to that expounded, in which the stronger eigenfunction dominates, still applies.

\section{REFERENCES}

1 Gdoutos, E. E. and Theocaris, P. S. Stress concentration at the apex of a plane indenter acting on an elastic half-plane. Trans. ASME, J. Appl. Mechanics, 1975, 42, 688-692.

2 Williams, M. L. Stress singularities resulting from various boundary conditions in angular corners of plates in extension. Trans. ASME, J. Appl. Mechanics, 1952, 19, 526-528.

3 Barber, J. R. Elasticity, 1992 (Kluwer, Dordrecht).

4 Filippi, S., Lazzarin, P. and Tovo, R. Development of some explicit formulas useful to describe elastic stress fields ahead of notches in plates. Int. J. Solids Structs, 2002, 39, 4543-4565.

5 Khadem, R. and O'Connor, J. J. Axial compression of an elastic circular cylinder in contact with two identical elastic half spaces. Int. J. Engng Sci., 1969, 7(8), 785-800.

6 Bogy, D. B. Edge-bonded dissimilar orthogonal elastic wedges under normal and shear loading, Trans. ASME, J. Appl. Mechanics, 1968, 35(90), 460-466. 